\title{
Eau et environnement. Tunisie et milieux
} méditerranéens.

ENS Lyon éditions, Collection Sociétés, Espaces temps. 2003, 208 pages.

Jean-Claude Bonnefont

\section{OpenEdition}

\section{Journals}

Édition électronique

URL : http://journals.openedition.org/rge/2330

DOI : $10.4000 /$ rge. 2330

ISSN : 2108-6478

Éditeur

Association des géographes de l'Est

Édition imprimée

Date de publication : 1 septembre 2003

ISSN : 0035-3213

\section{Référence électronique}

Jean-Claude Bonnefont, "Eau et environnement. Tunisie et milieux méditerranéens. ", Revue

Géographique de l'Est [En ligne], vol. 43 / 4 | 2003, mis en ligne le 24 novembre 2010, consulté le 25 septembre 2020. URL : http://journals.openedition.org/rge/2330 ; DOl : https://doi.org/10.4000/rge. 2330

Ce document a été généré automatiquement le 25 septembre 2020

Tous droits réservés 


\title{
Eau et environnement. Tunisie et milieux méditerranéens.
}

ENS Lyon éditions, Collection Sociétés, Espaces temps. 2003, 208 pages.

\author{
Jean-Claude Bonnefont
}

\section{RÉFÉRENCE}

Eau et environnement. Tunisie et milieux méditerranéens. ENS Lyon éditions, Collection Sociétés, Espaces temps. 2003, 208 pages.

1 Paul Arnould et Micheline Hotyat ont recueilli dans ce volume les actes du Colloque franco-tunisien tenu à Hammamet les 14 et 15 novembre 1998, formés de 14 contributions, dont 8 intéressent spécialement le territoire tunisien, tandis que les autres ont un caractère plus général ou s'appliquent à des espaces voisins d'Afrique, d'Algérie ou de France. Elles balaient tous les aspects géographiques qui vont de la climatologie à la biogéographie, en mettant l'accent sur les faits hydrologiques, qui sont la clé de toutes les interprétations dans un milieu semi-aride. Les thématiques abordées sont, comme l'écrit Pierre Pagney d'une manière très synthétique, «les faits de répartition inégale, spatiale et temporelles, les risques, les aléas, la vulnérabilité des sociétés, les questions de transfert et de rééquilibrage ». C'est ainsi que le thème de la semi-aridité est décliné dans des communications qui mettent principalement l'accent sur les valeurs extrêmes, plus significatives pour le géographe que les moyennes, en étudiant les années pluvieuses et les grandes sécheresses; que les ressources en eau sont analysées de manière à montrer les déséquilibres régionaux et les moyens d'y faire face ; que l'étude de l'écoulement et du ruissellement, pour aboutir à des données quantitatives, s'enrichit d'études expérimentales réalisées sur le territoire français; que les rapports du milieu avec la végétation sont examinés dans des travaux qui portent aussi bien sur les couverts forestiers que sur l'environnement d'une sebkha périurbaine. Le résultat de toutes ces recherches originales, produites par des 
chercheurs dont 10 travaillent en Tunisie et 7 en France, est présenté de manière claire, aisément lisible et renforcée par une illustration de qualité.

2 L'ensemble de ces travaux, dont la très forte unité est le fruit d'une collaboration de plusieurs années, conduit Pierre Pagney à des conclusions qu'il énumère fort utilement à la fin du volume. En replaçant l'espace tunisien à l'échelle zonale, on comprend mieux le contact qu'il présente entre l'aridité du sud et la relative humidité du nord, qui possède $80 \%$ des eaux de surface ; on comprend par la même occasion le jeu de bascule qui se produit entre l'influence plus souvent prédominante des tropiques secs et celle de la circulation polaire. Nous sommes, c'est évident, dans un pays où les conditions climatologiques commandent presque tout. Mais on ne peut s'en tenir à cette échelle zonale. Il faut compléter ces faits généraux par des études plus fines, menées à l'échelle régionale et locale: elles seules montreront bien les relations qui existent entre l'environnement et les politiques d'aménagement et peuvent déboucher sur les problèmes de géographie appliquée.

3 C'est précisément sur le changement des perspectives en matière de géographie appliquée que Pierre Pagney insiste le plus nettement. On ne peut plus se contenter ni d'études générales, ni d'études régionales et locales prises isolément: il convient d'envisager la cohérence qui doit exister entre les phénomènes d'échelle inférieure et ceux d'échelle supérieure, c'est-à-dire replacer les études régionales dans leur cadre zonal, le seul qui soit adapté pour bien comprendre les répercussions d'un aménagement régional et local sur les régions voisines. C'est en agissant ainsi qu'en matière d'eau, les phénomènes de concurrence apparaîtront au premier plan. Et cela obligera tout naturellement à intégrer l'ensemble des faits observés localement à l'échelle nationale, dans le cadre de la politique d'un état. On est ainsi conduit à poser la question du développement d'un pays comme la Tunisie, soumis à de si lourdes contraintes physiques. Il n'existe pas pour les pays en développement de recette miracle, aisément transposable de l'un à l'autre, et les méthodes applicables sont celles qui devront tenir compte des données naturelles incontournables. En ce sens, le volume présenté apporte, au-delà de sa contribution au développement tunisien, une leçon générale dont il convient de tenir compte dans toutes les parties du globe.

\section{AUTEUR}

\section{JEAN-CLAUDE BONNEFONT}

Université Nancy 2 